\title{
Evolución de los modelos docentes de futuros profesores de economía de secundaria, a través de la metáfora*
} Evolution of teaching models of prospective secondary
economics teachers, through the metaphor

\author{
Lucía Mellado(1), Juan Luis de la Montaña ${ }^{(2)}$ \\ María Rosa Luengo ${ }^{(2)}$ y María Luisa Bermejo ${ }^{(3)}$
}

(1) Dto. Economía de La Empresa y Contabilidad. UNED, Madrid (2) Dto. Didáctica Ciencias Sociales y de las Lenguas y Literaturas. Universidad de Extremadura, Badajoz (3) Dto. Psicología y Antropología. Universidad de Extremadura, Badajoz

Resumen: A través de un cuestionario de preguntas abiertas, este estudio analiza la evolución de las metáforas personales de una muestra de profesores en formación de la especialidad de Economía del Máster Universitario en Formación del Profesorado en Educación Secundaria, durante el curso 2012/2013. Para el análisis se han adaptado las cuatro categorías de Leavy, McSorley y Boté (2007): conductistas-transmisivas, cognitivas-constructivistas, situadas y autorreferenciadas.

Los resultados indican que la mayoría de los participantes fueron capaces de conceptualizar sus roles en términos de metáforas. Al comparar las metáforas, antes y después de las prácticas de enseñanza, se produce una evolución progresiva de las metáforas conductistas-transmisivas a cognitivas-constructivistas y situadas. Para los profesores en formación, la reflexión sobre sus propias metáforas, se ha mostrado como una buena herramienta para hacerlos conscientes de sus concepciones y de sus roles docentes, y para iniciar cambios en los mismos hacia modelos didácticos más centrados en el alumnado.

Palabras clave: Metáforas, formación del profesorado, educación secundaria, economía.

Abstract: Using the responses to open questions, this study analyzes the evolution of personal metaphors of a sample of prospective secondary economics teacher during their Master's degree course in Secondary Teacher Education during the 2012/2013 academic year. The study applied an adaptation of the four categories of Leavy, McSorley \& Boté (2007): transmissive/ behavioral, cognitive/constructivist, situated and self-referential.

The results indicate that most of the prospective teachers were able to conceptualize their roles in the form of metaphors. Comparing metaphors before and after teaching practice, a progressive evolution occurs from transmissive-constructivist metaphors to cognitive-behavioral and situated metaphors. For preservice teachers, reflecting on their own metaphors, it has proven to be a good tool to make them aware of their conceptions and their teaching roles, and to initiate changes in them towards more focused teaching models in students

Keywords: Metaphors, teacher education, secondary education, economics.

(Fecha de recepción: noviembre, 2015, y de aceptación: marzo, 2016)

DOI: 10.7203/DCES.30.7230

* Agradecimientos: Este trabajo ha sido financiado por el proyecto de investigación EDU2012-34140 del Ministerio de Economía y Competitividad y el Gobierno de Extremadura. 


\section{Introducción}

Como señalan Travé y Molina (2014) la incorporación de la economía a la educación obligatoria requiere configurar una didáctica de la economía, dentro de la didáctica de las ciencias sociales, que fundamente la formación inicial y continua de estos profesores. La alfabetización económica de los estudiantes es fundamental para procurar a los futuros ciudadanos recursos esenciales con los que participar activamente en la construcción de sociedades modernas y democráticas (Cañas y de la Montaña, 2012).

Desde la década de los noventa se han desarrollado distintas líneas de investigación en didáctica de la economía siendo una de ellas el estudio de las concepciones y la práctica del profesorado de secundaria de economía, tanto en formación como en servicio (Travé, 1998). La comprensión de los factores que favorecen u obstaculizan la formación y el desarrollo profesional del profesorado es actualmente uno de los temas más relevantes de la agenda de la investigación educativa y un elemento esencial para planificar y llevar a cabo programas de formación que tengan como resultado una mejora de la enseñanza y del aprendizaje en las aulas (Estepa y Cuenca, 2007; Hargreaves, 1996; Mellado et al., 2006). Con la implantación de la especialidad de Economía en el Máster Universitario en Formación del Profesorado en Educación Secundaria (MFPS) se ha dado un importante paso en la formación y la identidad profesional de este profesorado.

Sin embargo, cuando los futuros profesores de economía se incorporan al Máster, lo hacen no sólo con un bagaje de conocimientos, sino con unos valores, concepciones, roles y actitudes sobre la enseñanza, el aprendizaje y el rol del profesor, fruto de los muchos años de su propia escolaridad. Estas concepciones didácticas personales, adquiridas de forma natural y sin reflexión de sus propias experiencias como estudiantes, están profundamente arraigadas y son difíciles de cambiar (Gallego y Pérez, 2002). Esta impregnación hace que muchos profesores utilicen en la práctica los métodos didácticos que ellos mismos preferían como estudiantes, o simplemente enseñan de la misma forma en que fueron enseñados (Sillmam y Dana, 2001; Tobin et al., 1994). Por tanto un primer paso en la formación es que los profesores analicen sobre sus propias concepciones y modelos didácticos, sean conscientes de los mismos y de las posibles alternativas y tengan los conocimientos y herramientas necesarias para controlar y autorregular los cambios en los mismos.

En el presente artículo presentamos una investigación en la que analizamos a través de las metáforas personales, la evolución de las concepciones sobre el rol del profesor, de un grupo de futuros profesores de economía del MFPS, antes y después de las prácticas de enseñanza. El trabajo forma parte de una investigación más amplia realizada por un equipo interdisciplinar 
de distintas didácticas específicas y de psicología. La interdisciplinariedad en un trabajo de esta naturaleza es una necesidad por el objeto de estudio, pero también una oportunidad de abrir nuevos horizontes por la diferente perspectiva de cada área.

\section{Las metáforas personales del profesorado}

Cada profesor elabora un pensamiento práctico profesional idiosincrásico, a partir de su experiencia personal y de la interacción social con el entorno que le rodea. A este pensamiento es difícil acceder y dotar de significados, ya que éste tiene unas percepciones sobre la enseñanza y el aprendizaje, que les resultan difíciles de articular y de expresar de una forma estructurada. El lenguaje que utiliza el docente para hablar de sus concepciones, roles y de su actividad profesional no suele ser literal y estructurado, sino más bien simbólico y de carácter metafórico (Lakoff y Johnson, 1986).

Una metáfora es la sustitución o transposición de una idea o concepto por otro que tenga con él una cierta relación de semejanza objetiva o subjetiva para el emisor (Marcos, 1993). Para Lakoff y Johnson (1986) lo esencial de la metáfora es comprender y experimentar una cosa en términos de otra. Las metáforas se han utilizado como un recurso expresivo en la literatura, en la oratoria, en la música y en numerosas expresiones artísticas y de comunicación. Desde la psicolingüística se estudia la correla- ción entre comportamiento lingüístico y procesos psicológicos subyacentes por lo que la metáfora sería un mecanismo heurístico que se manifiesta lingüísticamente. Para De Bustos, (2000) las metáforas lingüísticas serían sólo la punta del iceberg de procesos cognitivos más básicos. En lingüística cognitiva, la metáfora es definida como un mecanismo cognitivo básico que consiste en la proyección de un dominio de conocimiento concreto, más conocido o estructurado, a otro dominio generalmente más abstracto y menos estructurado lingüísticamente (Alarcón y Díaz, 2014)

Las metáforas, por tanto, no son sólo un recurso expresivo, sino que tienen un significado más profundo ya que constituyen un mecanismo esencial de la mente, que estructura gran parte del sistema conceptual por medio de relaciones metafóricas y son un principio fundamental del pensamiento y la acción (Lakoff y Johnson, 1986). Las metáforas son como lentes, pantallas o filtros de lo que somos y de cómo nos vemos en determinada situación (Saban, 2010). Para Duffé (2004) las metáforas actúan como un código de valores compartidos por otros miembros de la comunidad cultural y lingüística y constituyen un medio de comprender el mundo. La metáfora es esencial a la comprensión humana y un mecanismo para crear nuevo significado y nuevas realidades en nuestras vidas. A través de las metáforas cada individuo es capaz de construir conceptos abstractos a partir de imágenes esquemáticas y de conceptos 
directamente ligados a la experiencia (Lakoff y Johnson, 1986).

$\mathrm{Al}$ ser un componente esencial del razonamiento, la argumentación y la inferencia científica (De Bustos, 2000), las metáforas están presentes en el contexto del descubrimiento científico y en el contexto de su justificación y comunicación (Willison y Taylor, 2006). En economía el estudio de las metáforas es un campo aceptado de investigación (Herrera-Soler y White, 2012), y aunque no es objeto de este trabajo, señalar que las metáforas forman parte de las teorías económicas (Fukuda, 2009), de la teoría de las organizaciones (Morgan, 1980), de las finanzas y contabilidad (Walters y Young, 2008), del mercado (Teichert et al., 2006), de lo comercial (Madhavaram y McDonald, 2010), de los empresarios (Dodd, 2012) y de los recursos humanos (McCorkle y Gayle, 2003).

Para el profesorado las metáforas son un medio para articular el pensamiento y para establecer puentes entre las concepciones y la narrativa que describe la vida del aula (Ben-Peretz et al., 2003; Buaraphan, 2011; René y Stofflett, 1996; Saban et al., 2007; Volkmann y Anderson, 1998; Zhao et al., 2011), ya que permiten averiguar los referentes implícitos que sustentan las concepciones, dan una visión holística de la vida del aula e influyen en la conducta docente de los profesores (BouJaoude, 2000; Gurney, 1995; Tobin y LaMaster, 1995). Un importante aspecto para la educación, señalado por Lakoff y Johnson (1986) es que las metáforas pueden crear realidades sociales y convertirse en guía para las acciones futuras que se ajustarán a la metáfora "las metáforas pueden ser profecías que se cumplen" (p.198). En este sentido numerosos trabajos han mostrado que los profesores realizan cambios en sus concepciones y en su práctica en el aula cuando son capaces de construir nuevas metáforas compatibles con tales cambios (Martínez et al., 2001; Pinnegar et al., 2011; Russell y Hrycenko, 2006; Thomas y Beauchamp, 2011; Tobin et al., 1994).

El estudio de las metáforas ha sido defendido como una potente herramienta que estimula y potencia la reflexión del profesorado (Paavola y Hakkarainen, 2005). El pensamiento metafórico ayuda a los profesores a reflexionar sobre sus concepciones, sus roles y su práctica, y a reconceptualizarlos en un proceso de autorregulación metacognitiva (Aubusson et al., 2006). Además las metáforas tienen un fuerte componente afectivo y pueden ayudar a los profesores a tomar conciencia de sus sentimientos y emociones (Mellado et al., 2014a y 2015; Rebollo et al., 2008 y 2013, Tobin y Tippins, 1996).

Desde la década de los 90 contamos con importantes estudios sobre las metáforas de los profesores (Bullough, 1991; Ritchie, 1994; Tobin et al., 1994). Sin embargo coincidimos con Shaw et al. (2008) en que las metáforas deben seguir siendo objeto de investigación en aspectos menos tratados, como su incidencia en el cambio de los modelos didácticos. En particular nos parece fundamental que los profesores anali- 
cen sus metáforas personales, antes y después de las prácticas, pues las metáforas usadas durante las prácticas son un catalizador para la reflexión y pueden ayudar al profesorado a comprender y autorregular sus roles (Gosselin y Meixner, 2015; Seung et al., 2011; Tannehill y MacPhail, 2014), así como los obstáculos a cambiar sus metáforas, que están en gran parte determinadas por sus largos años de escolaridad como alumnos (Mahlios et al., 2010).

\section{Problema de investigación}

Este trabajo forma parte de una investigación más amplia realizada por un equipo interdisciplinar de distintas didácticas específicas y de psicología, que analizan las metáforas personales del profesorado en formación de infantil, primaria y secundaria de distintas especialidades sobre la enseñanza y el aprendizaje, su relación con las emociones y su evolución antes y después de las prácticas de enseñanza.

Los objetivos de este estudio son:

1. Averiguar las metáforas personales sobre el rol del profesor de una muestra de futuros profesores de secundaria de la especialidad de Economía del Máster Universitario en Formación del Profesorado en Educación Secundaria.

2. Conocer las razones y explicaciones que aportan para identificarse con tales metáforas.

3. Clasificar las metáforas personales de la muestra dentro de las catego- rías: conductista-transmisiva, cognitiva-constructivista, situadas y autoreferenciadas.

4. Analizar la evolución de las metáforas antes y después de las prácticas de enseñanza.

\section{Metodología}

La muestra la componen los futuros profesores de secundaria que realizaron el Máster Universitario en Formación del Profesorado en Educación Secundaria en la Universidad de Extremadura, durante el curso 2012-2013 en la especialidad de Economía. Durante el Máster se les informó de los objetivos del estudio y de la voluntariedad de participar en el mismo. El grupo de Economía estaba formado por 26 sujetos en el pretest ( 6 hombres y 20 mujeres) y por 17 en el postest ( 3 hombres y 14 mujeres). El menor número de participantes en el postest, fue por la dificultad de reunirlos después de la realización de las Prácticas de Enseñanza. Como en otras carreras de sociales en la muestra hay predominio de mujeres, lo que refleja la feminización de la profesión y quizás también la relación entre la elección de la carrera y los estereotipos de género asociados a la profesión (Gutiérrez y Luengo, 2003). La muestra se ha elegido por conveniencia y no pretende ser representativa en términos estadísticos, sino relevante para el estudio realizado.

El procedimiento de recogida de datos ha sido un cuestionario anónimo en el que además del sexo, la edad 
y los antecedentes académicos se le hacen preguntas abiertas sobre sus propias metáforas sobre la enseñanza, así como las razones que les llevan a identificarse con esas metáforas. También se incluyeron preguntas sobre las metáforas con las que identifican el aprendizaje del alumnado, aunque este aspecto no lo analizaremos en este artículo. Para elaborar el cuestionario se tomaron como referencia las investigaciones previas de Leavy et al. (2007) y Martinez et al. (2001) y nuestros propios estudios anteriores (Mellado et al., 2012), en los que fue validado el cuestionario abierto. Los procedimientos de recogida de datos incluyen también la realización de dibujos sobre las metáforas, instrumentos validados en anteriores estudios de con profesores en formación (Buaraphan, 2011; Mellado et al., 2014b). Antes de pasar el cuestionario se les informó sobre el significado y la importancia de las metáforas en educación, pero no se les dio ningún ejemplo de metáforas personales para no condicionar sus respuestas.

Las preguntas del cuestionario que analizamos en este artículo fueron:

1. Cuando impartes docencia en un aula, ¿Con que metáfora o metáforas te identificas como profesor o profesora?

2. Explica las razones por las que te identificas con esas metáforas

3. Por favor, intenta hacer un dibujo que represente tus metáforas como profesor/a y la relación con el proceso de aprendizaje de tus alumnos
En nuestro estudio adaptamos las cuatro categorías de Leavy et al. (2007): la conductista-transmisiva: el alumno como un aprendiz pasivo, el profesor como un transmisor de información y la motivación dependiente de refuerzos externos, que tiene además la característica de ser una clase centrada en el profesor y el contenido; la cognitiva-constructivista: el alumno como un agente activo en el proceso de construcción de su propio conocimiento y el profesor como un facilitador del proceso; la situada o de aprendizaje social: el aprendizaje es situado en el contexto social y la motivación surge del compromiso con la comunidad educativa, estando con la cognitiva-constructivista centrada en el alumno y el aprendizaje; la autorreferenciada, que son metáforas de difícil clasificación y que tienen un componente egocéntrico, cuyo significado es necesario analizar en cada caso.

Hemos incluido también algunos proverbios, sintagmas convencionales o modismos expresados por los profesores. Los modismos son frases cortas y fijas con sentido privativo, cuyo significado conjunto está asumido por el colectivo cultural y lingüístico, pero que no se deduce literalmente de las palabras que lo forman. Para Gibbs, 1992 (citado por Duffé, 2004) los modismos son como "metáforas cristalizadas" que encierran significados muy arraigados en una comunidad.

Se ha procedido a un análisis descriptivo de frecuencias en cada grupo y categoría y han discutido las razones 
por las que identifican con las metáforas, contrastando los resultados con anteriores estudios, así como entre las metáforas escritas y los dibujos que las representaban.

Finalizado el análisis del pretest, y antes de las prácticas de enseñanza, se realizó una sesión con el grupo en el que se les expusieron en power point las metáforas y los dibujos que las representaban y se discutieron sus significados. El postest lo realizaron después de las prácticas de enseñanza, aunque ya no había sesiones regladas de las asignaturas del Máster y la recogida de datos se realizó en una de las sesiones de tutoría sobre las prácticas.

\section{Discusión de los resultados}

$\mathrm{Al}$ ser una muestra no representativa estadísticamente, los resultados no pretenden ser generalizables, sino más bien, desde una perspectiva interpretativa, buscar los significados de las metáforas individuales de los participantes. Hemos identificado a los participantes que han realizado tanto el pretest como el postest, por el código anónimo personal que incluyeron en el pre y postest, manteniendo en todo caso el anonimato. Esta identificación nos permite seguir su evolución, antes y después de las Prácticas de Enseñanza. De ello se obtiene que 12 participantes sólo realizaron el pretest, 3 sólo realizaron el postest y 14 realizaron ambos.

En el pretest hemos identificado 41 metáforas para el rol del profesor en los 26 participantes en el mismo. En el pos- test hemos identificado 22 metáforas para el rol del profesor en los 17 que lo realizaron. La mayoría de metáforas del profesor del pretest son transmisivasconductistas (un 60.9\%), seguidas de las cognitivas-constructivistas (19.5\%), y de las autorreferenciadas y situadas (9.8\%). En cambio en el postest hay el mismo número de metáforas del profesor conductistas transmisivas que cognitivas-constructivistas $(31.8 \%)$, seguidas de las situadas $(27.3 \%)$ y de las autorreferenciadas (9.1\%).

$\mathrm{Al}$ comparar en el gráfico I las metáforas para el profesor, antes y después de las Prácticas de Enseñanza (pretest y postest) se observa una gran disminución de las metáforas conductistastransmisivas y un aumento de las cognitivas-constructivistas y de las situadas, manteniéndose un porcentaje similar para las autorreferenciadas. Sin embargo hay que tener en cuenta que el pretest lo realizaron 9 profesores más que el postest, por lo que el estudio de la evolución de las metáforas será más significativo en los 14 participantes que realizaron ambos cuestionarios, como trataremos más adelante.

Comentaremos en primer lugar algunas de las metáforas expresadas por los que sólo realizaron o el pretest 0 el postest, para a continuación analizar la evolución de las metáforas de los 14 participantes que realizaron el pre y el postest.

Entre las transmisivas-conductistas para el profesor se repite varias veces la del libro abierto, con el significado de centrar el modelo de enseñanza en la 


\section{Gráfico I}

Comparación del porcentaje de metáforas para el profesor del grupo de Economía, antes y después de las Prácticas de Enseñanza (pretest y postest)

\section{Metáforas Economía Profesor}

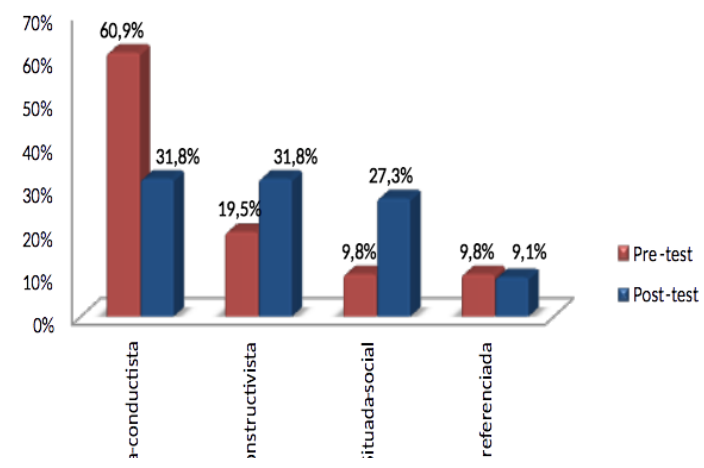

explicación del profesor, tan clara como un libro abierto, metáfora señalada por varios autores (Alarcón et al., 2014; Thomas y Beauchamp, 2011; Sillmam y Dana, 2001) y también encontrada en otra muestra de futuros profesores de economía (Mellado et al., 2012). Otro se ve como google, que lo sabe todo y tiene que transferir sus conocimientos a los alumnos, a los que ve como un depósito vacio. La clase centrada en el profesor se muestra también en las metáforas del Sol (Seferoglu et al., 2009) o del líder.

Con el mismo significado de la explicación del profesor, otro participante señala la metáfora de clara como el agua, también encontrada en nuestro estudio anterior en un futuro profesor de economía (Mellado et al., 2012). Otro se ve como si se hubiera tragado un megáfono para expresar lo alto que habla, o la del rayo de este mismo profesor para expresar la rapidez en su explicación.
El dibujo de la figura I de uno de los participantes muestra perfectamente estas características, con una profesora en la pizarra concentrada en su propia explicación (López-Luengo et al., 2015), y los alumnos distraídos hablando entre sí y sin prestar atención a la explicación de la profesora.

Figura I

Dibujo de la profesora explicando a alumnos distraídos

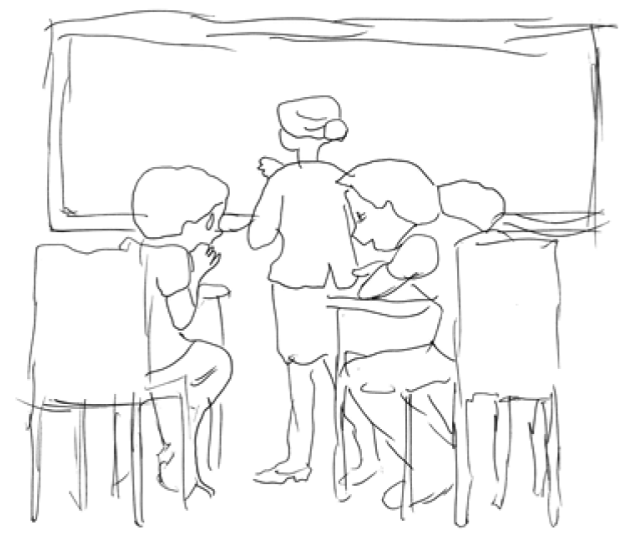


Un participante se identifica como profesor con la metáfora conductista del juez que imparte justicia a todos por igual, metáfora señalada por BenPeretz et al. (2003) y Lorsbach et al. (1992). Algunas de las metáforas conductistas se identifican con el profesor autoritario, duro como una piedra. En los dibujos realizados por uno de los participante se representa como un ángel: "por las buenas puede ser un sol”, o como un demonio: "por las malas su peor pesadilla”. Estas metáforas expresan emociones negativas muy fuertes. Las metáforas tienen un fuerte componente afectivo y pueden ayudar a los profesores a tomar conciencia de sus sentimientos y emociones (Mellado et al., 2014a y 2015; Rebollo et al., 2008 y 2013).

Varias metáforas cognitivas constructivistas para el profesor (la esponja, el tren, la mano y el sol, ascender la montaña, el sembrador, la luz y el calor y el árbol) son comunes entre el pretest y el postest, por lo que las comentaremos posteriormente.

Entre las situadas para el profesor se encuentra la del pájaro que es libre y guía a sus alumnos y mira desde arriba con cierta perspectiva (Thomas y Beauchamp, 2011). Otras metáforas situadas para el profesor, que comentaremos posteriormente, son la de la batuta del director de orquesta, la del camino y la de la luz que ilumina el camino.

Entre las autorreferenciadas para el profesor hemos identificado varias de animales, como la hormiga o el búho.
Los participantes del grupo de Economía no han expresado metáforas relacionadas con la economía ni con los contenidos específicos de sus especialidades formativas. Este resultado coincide con otros anteriores (Mellado et al., 2012), en que las metáforas no está asociadas a contenidos concretos, sino que son expresión de una visión general de la enseñanza y del rol del profesor, formada de sus propias experiencias como alumnos y de sus experiencias de enseñanza.

Evolución de las metáforas en los participantes de Economía que realizaron el pretest y el postest

Hubo 14 participantes que realizaron tanto el pretest como el postest, por lo que en estos futuros profesores puede realizarse un seguimiento de la evolución de sus metáforas durante la realización del Máster, antes y después de las prácticas de enseñanza en los centros de secundaria. A continuación analizaremos la evolución de las metáforas de estos 14 participantes, que identificaremos con las siglas E1, E2... E14.

Los participantes E1 y E2 mantienen sus metáforas conductistas-transmisivas, para el profesor, antes y después de las prácticas de enseñanza. E1 representa al profesor como una máquina, tanto en el pre como en el postest (figura II), manteniendo la misma metáfora durante el Máster. La metáfora de la máquina es habitual en los estudios sobre las organizaciones empresariales (Morgan, 1980), representando una 
visión tradicionalista de la empresa, trabajando como máquinas, de forma rutinaria, eficiente, exacta y predeci- ble. También Weiner (1991) utiliza esta metáfora en su teoría de la motivación y de la atribución de causas.

\section{Figura II}

\section{Dibujos para la metáfora de la máquina del profesor (pre y postest)}

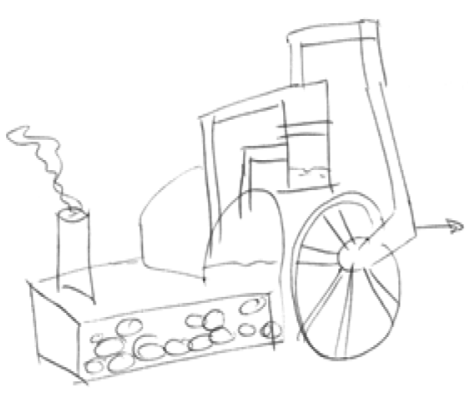

El participante que denominamos E2 mantiene sus metáforas conductistastransmisivas. Para el profesor se identifica con la mirada asesina (antes) y

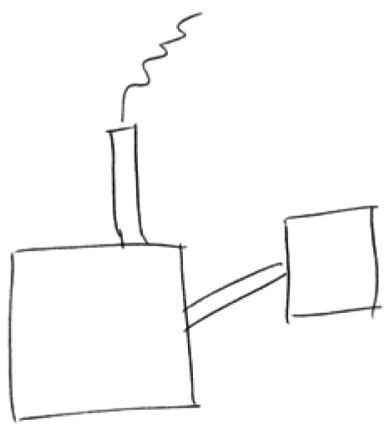

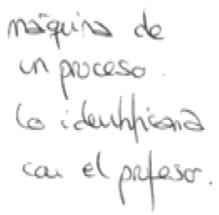

la mirada del lobo (después), metáforas que tienen un alto grado de agresividad (figura III) y que expresan emociones negativas muy fuertes.

\section{Figura III}

Dibujos para la metáfora de la mirada asesina y mirada de lobo del profesor (pre y postest)

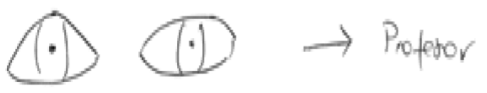

El participante E3 cambia sus metáforas, pero las mantiene dentro de las conductistas-transmisivas. En el pretest se identifica con el futbolista Cristiano Ronaldo, que se caracteriza más por ser una figura centrada en el juego individual que en repartir juego a sus compañeros, y del lobo entre corderos. En el dibujo del pretest se representa como una boca que transfiere el cono-

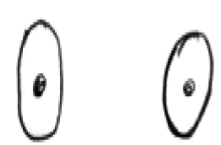

Protesol cimiento a los alumnos-esponjas. En el postest se representa como un libro abierto (figura IV). Este futuro profesor representa metáforas diferentes en las escritas que en las dibujadas. Las metáforas del texto expresan cualidades y emociones individuales del profesor, mientras que los dibujos se refieren al modelo transmisivo de enseñanza del profesor y del libro a los alumnos. 


\section{Figura IV}

\section{Dibujos para la metáforas conductistas-transmisivas del profesor y los alumnos (pre y postest)}

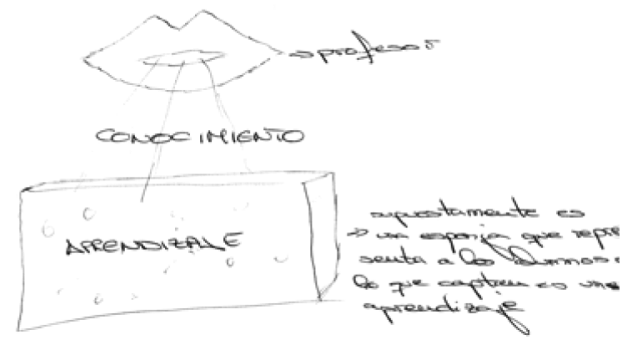

E4 se mantiene dentro de la categoría conductista-transmisiva, pero cambia la metáfora del profesor del libro abierto por el pozo de sabiduría y la luz

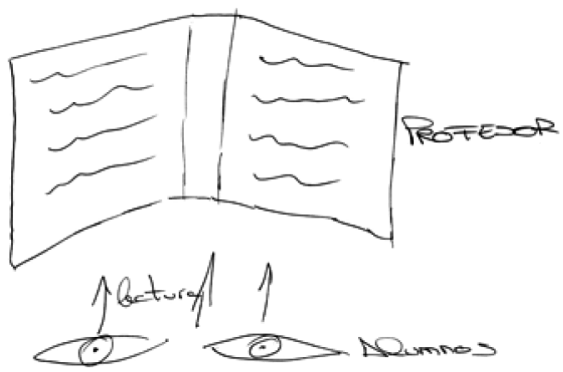

de la ciencia. Los dibujos son coherentes con este modelo: el del pretest representa un libro y el del postest al profesor dirigiéndose a los alumnos (figura $\mathrm{V}$ ).

\section{Figura V \\ Dibujos para la metáfora del libro abierto y del pozo de sabiduría del profesor (pre y postest)}

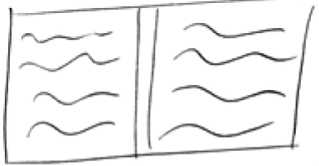

Profesores

E5 también mantiene sus metáforas antes y después de las prácticas, dentro de la categoría cognitiva-constructivista. Tanto en el pre como en el postest se identifica con el sembrador y a los alumnos con las semillas que germinan y crecen (figura VI). El sembrador tiene que regar y cuidar a todas las plantas según sus necesidades. La metáfora del sembrador ha sido identificada en varios trabajos como cognitiva constructiva (Buaraphan, 2011), complementaria de la metáfora de la semilla

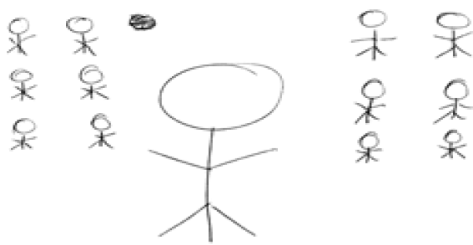

para el alumno (Gurney, 1995; Saban, 2010). Seung et al. (2011) además relacionan estas metáforas con mantener un buen clima en el aula.

La metáfora del desarrollo de las plantas para el proceso de enseñanzaaprendizaje la mantiene el participante E6 en el pre y postest. En ambos casos las hemos catalogado como cognitivasconstructivistas, ya que el profesor y los demás componentes de la educación serían los rayos del sol, que aportan lo que los seres vivos necesitan para su 
Figura VI

Dibujos para la metáfora del sembrador para el profesor y de la semilla para los alumnos (pre y postest)

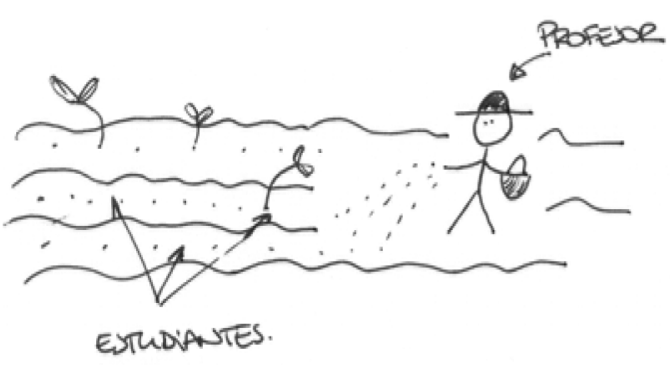

desarrollo (figura VII), pero el profesor no está solo sino en el contexto de la comunidad educativa. Las metáforas orgánicas se clasifican como cognitivasconstructivista y reflejan organismos

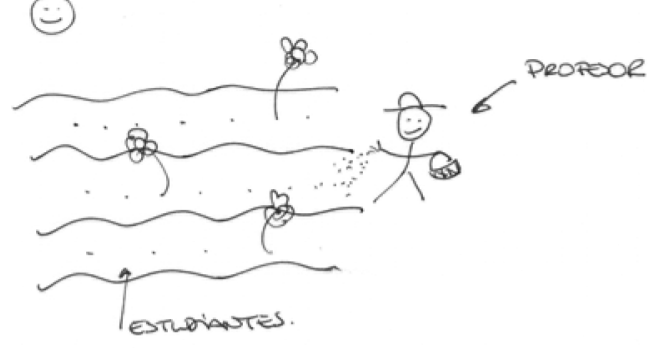

que crecen y se desarrollan (Saban, 2010) incidiendo más en el proceso del aprendizaje que en el producto final Cassel y Vincent (2011).

\section{Figura VII}

Dibujos para la metáfora del desarrollo de los seres vivos (pretest y postest)

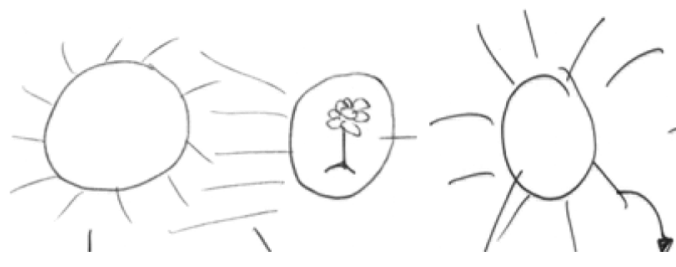

El participante E7, que básicamente mantiene para el profesor metáforas situadas, en el pretest se identifica con
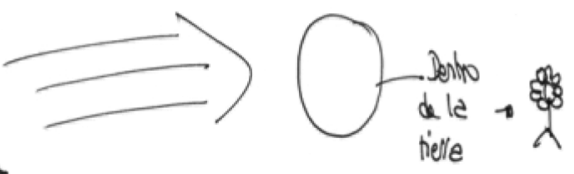

el camino o mano que guían, y en el postest el camino a seguir, que guía a los alumnos por el laberinto (figura VIII).

Figura VIII

Dibujos para las metáforas de la mano que guía (pretest) y del camino a seguir (postest)
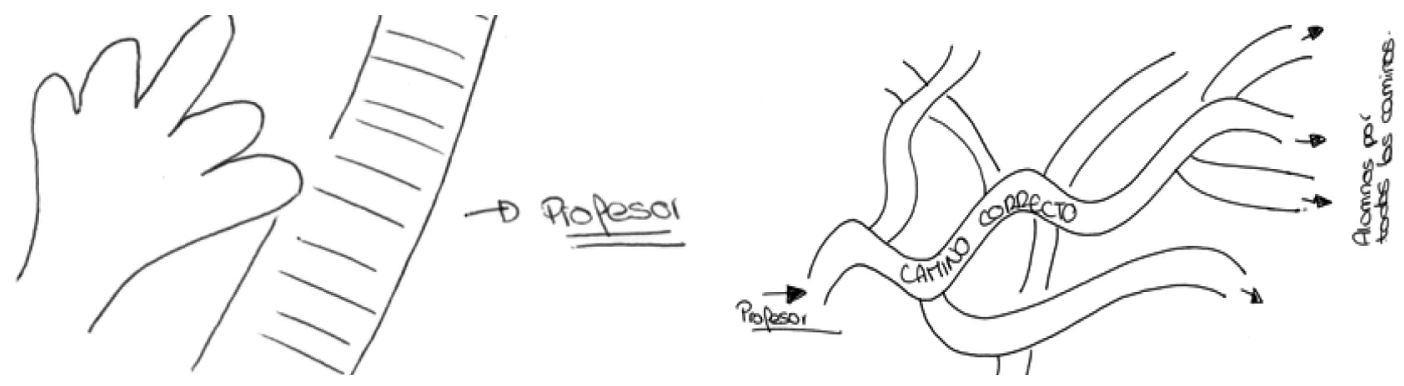
E8 mantiene la metáfora del director de orquesta (figura IX), antes y después de las prácticas de enseñanza. En otros trabajos esta metáfora ha sido catalogada como cognitiva-constructivista (López-Luengo et al., 2015) o como situada, porque el director dirige a un colectivo, pero cada alumno toca un instrumento diferente (Ben-Peretz et al., 2003; Levy et al., 3007; Seferoglu et al., 2009; Serrano y Pozo, 2006), que coincide con el significado dado por este participante, tanto en el texto como en los dibujos.

\section{Figura IX}

\section{Dibujos para la metáfora del director de orquesta (pre y postest)}

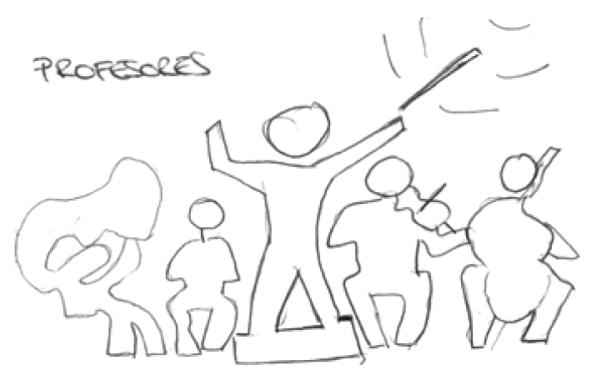

En E9 se mantienen sus metáforas situadas sobre el profesor: luz que ilumina el camino (pretest) y que los ilu-

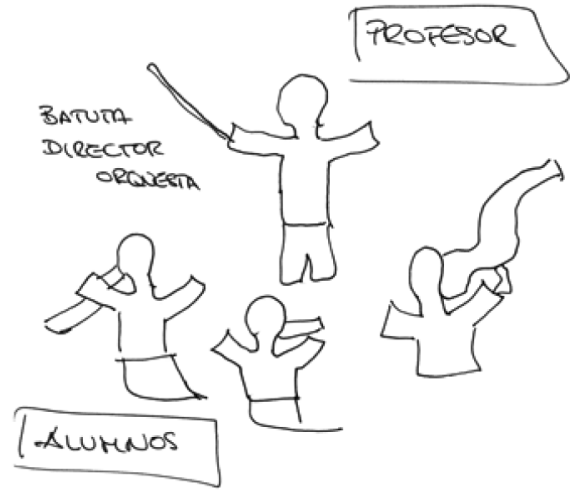

mina y los guía en el postest, mostrando también coherencia en lo expresado en el texto y en los dibujos (figura $\mathrm{X}$ ).

\section{Figura $X$}

Dibujos para las metáforas de luz que ilumina el camino del profesor (pretest y postest)

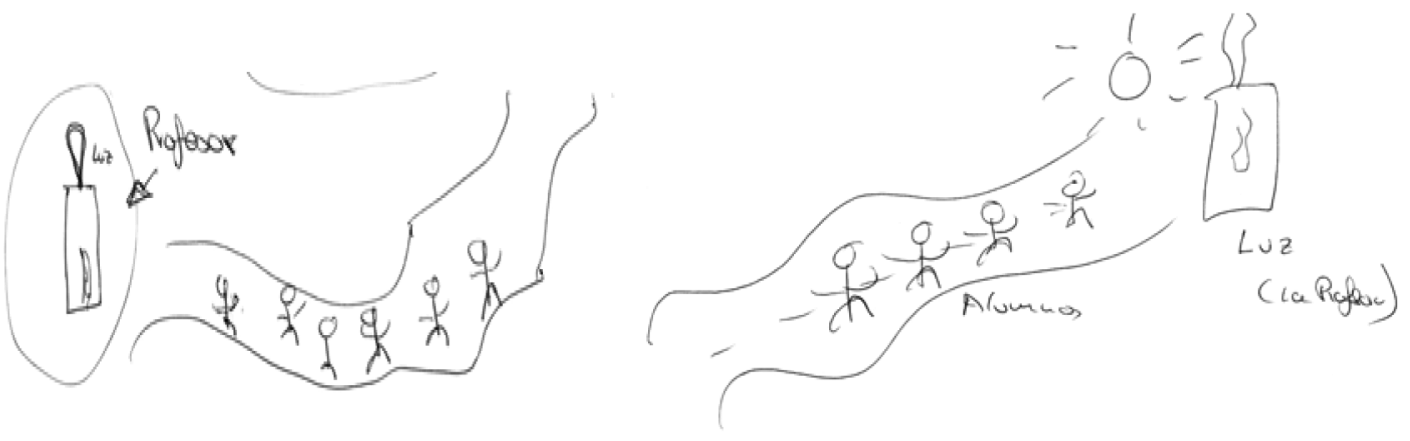


En E10 se detecta una evolución en sus metáforas del profesor. En el pretest se identifica con un tren que tiene que llegar a una hora a las estaciones y que avanza con vagones-alumnos o sin ellos, lo que denota una imagen conductista-transmisiva, porque el tren va a su ritmo, independientemente de las características de los vagones-alumnos y el vagón que no lo pueda seguir se desengancha y no llega a la meta (para esta metáfora no ha realizado dibujo). Para Saban (2010) la metáfora del vagón de tren representa la sumisión del alumno al profesor, ya que éstos tienen un rol pasivo supeditados a la máquina. En el postest cambia la metáfora y considera al profesor como la luz que guía el camino (figura XI), identificada como situada en numerosos trabajos (Gurney, 1995; Sillman y Dana, 2001; Thomas y Beauchamp, 2011).

E11 en el pretest se identifica con la metáfora de la hormiga, por ser muy trabajadora, que inicialmente clasificamos como autorreferenciada, pero

\section{Figura XI}

Dibujos para la metáfora de luz en el camino para el profesor (postest)

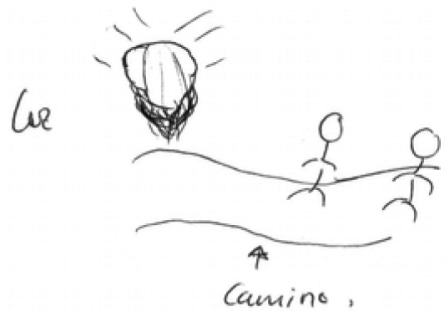

que en el dibujo (figura XII) se muestra escribiendo en la pizarra sin participación de los alumnos, por lo que tiene connotaciones transmisivas. En el pretest también se identifica con la metáfora de la esponja para el profesor, mucho más habitual para alumnos, con el significado de estar siempre aprendiendo (Crawford, 2000). Sin embargo en el postest las metáforas que señala para el profesor son las del sembrador, de características cognitivas-constructivistas, y del perro guía como lazarillo, que puede clasificarse entre situada, por ser guía, y cognitiva-constructi-

\section{Figura XII}

\section{Dibujos para la metáfora del profesor como hormiga (pretest)}

\section{y como sembrador y perro guía (postest)}
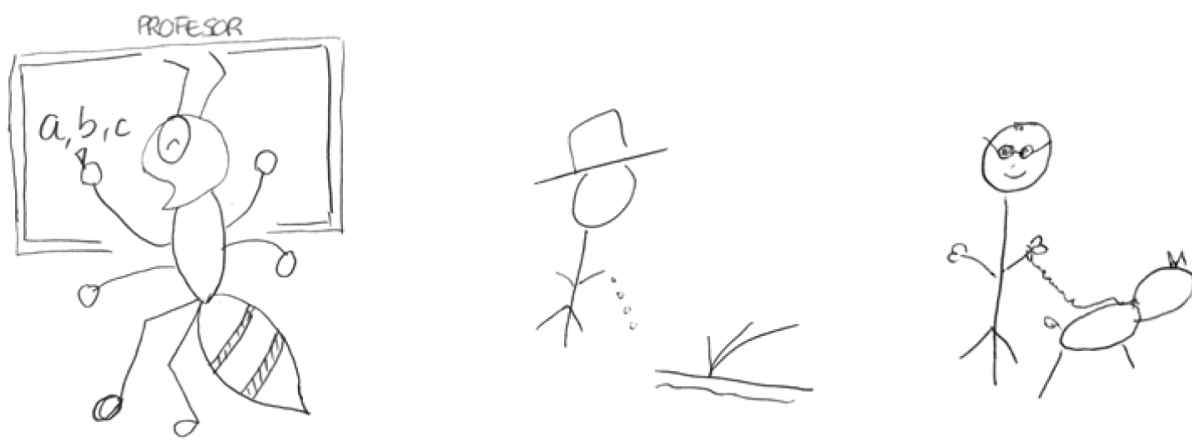

DIDÁCTICA DE LAS CIENCIAS EXPERIMENTALES Y SOCIALES. N. 30. 2016/1,23-44 
vista, por ser una ayuda para el ciegoalumno, que para este futuro profesor es el que toma sus propias decisiones. Estas metáforas muestran una clara evolución en este participante antes y después de las prácticas.
También se detecta evolución en las metáforas del profesor de E12 que pasa de la metáfora conductista del líder en el pretest, hacia otra más situadas, como el camino a seguir, en el postest (figura XIII).

\section{Figura XIII \\ Dibujos para las metáforas del profesor del líder (pretest) y del camino (postest)}

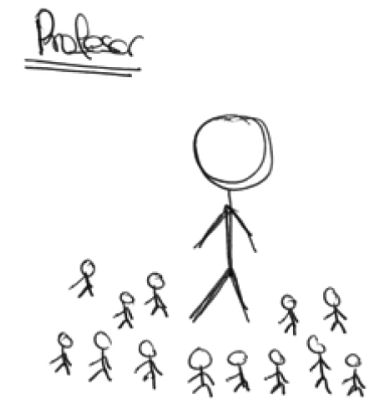

También hemos detectado una evolución en E13. En el pretest se identifica con la metáfora del padre (conductista), que representa la autoridad y el respeto, pero también el cariño y el cuidado (cognitiva), metáfora señalada en numerosos estudios previos (Alarcón et al., 2014; Buaraphan, 2011; Seferoglu et al., 2009); también se identifica

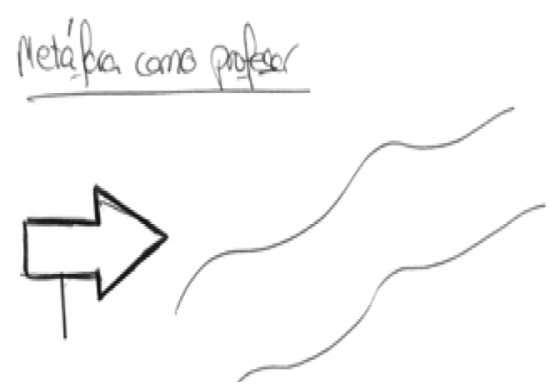

con la metáfora del amigo, cognitiva por la cercanía; y con la del búho (autorreferenciada), por la observación y la sabiduría, señalada por Saban (2010) (figura XIV). En el postest se identifica con el sembrador y para el alumnado en las plantas que crecen y se desarrollan, metáforas orgánicas de significado cognitivo-constructivista.

\section{Figura XIV}

Dibujos para las metáforas de padre, amigo y búho para el profesor

(pretest) y del sembrador (postest)
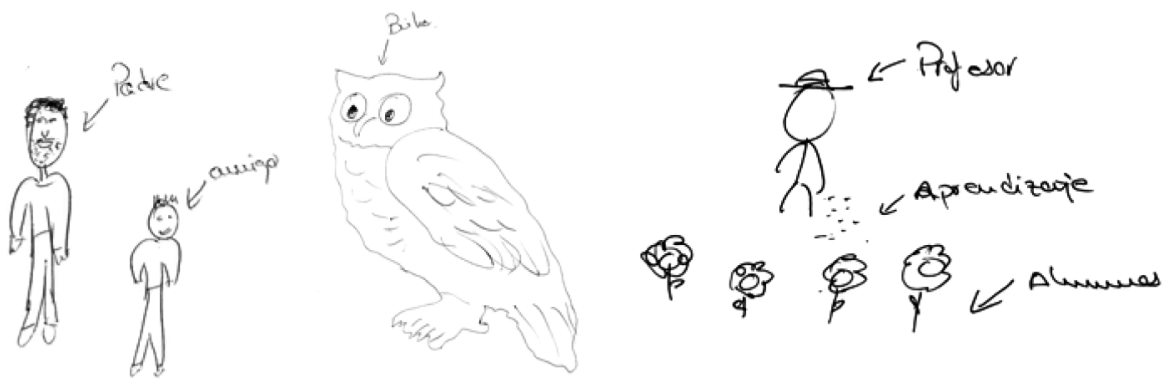
Finalmente E14 muestra también una progresión en el significado de sus metáforas. En el pretest se identifica con la metáfora de ascender una montaña con trabajo y sudor (cognitiva-constructivista) (figura XV). En el postest incide en el trabajo bien hecho y en el arte de enseñar, pero añade que es el guía de la escalada (situada) y el propio dibujo representa a varias figuras. Escalar una montaña es una metáfora identificada por Cassel y Vincent (2011) que incide en la meta final (producto), pero también en la importancia de la propia escalada (proceso).

\section{Figura XV}

\section{Dibujos para la metáfora de escalar una montaña (pre y postest)}

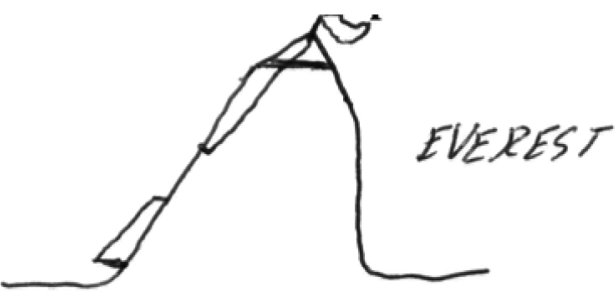

En la tabla I se resumen los cambios realizados antes y después de las prácticas de enseñanza para los 14 participantes que realizaron el pre y el postest: 8 no cambiaron de categoría en sus

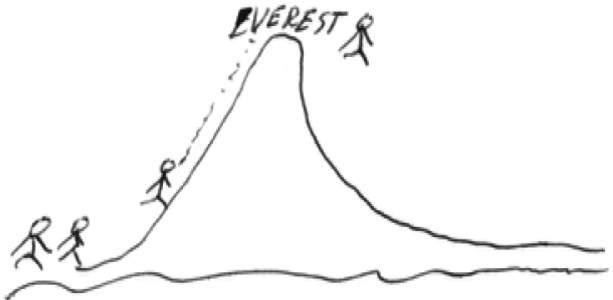

metáforas, aunque alguno cambió las metáforas dentro de la misma categoría y cinco cambiaron sus metáforas del profesor, realizando una progresión en los modelos.

\section{Tabla I}

Cambios en las metáforas del profesor antes y después de las Prácticas de Enseñanza

( $\mathrm{T}$ = Transmisiva-conductista $; \mathrm{C}=$ Cognitiva-Constructivista; $\mathrm{S}=$ Situada-social; $\mathrm{A}=$ Auto-referenciada)

\begin{tabular}{|c|c|c|}
\hline $\begin{array}{c}\text { Participantes comunes } \\
\text { al pre y postest }\end{array}$ & $\begin{array}{c}\text { Evolución metáforas profesor } \\
\text { del pre al postest }\end{array}$ & $\begin{array}{c}\text { Cambios de categoría en las } \\
\text { metáforas del profesor }\end{array}$ \\
\hline E1 & T --- T & No cambian \\
\hline E2 & T --- T & No cambian \\
\hline E3 & T --- T & No cambian \\
\hline E4 & T --- T & No cambian \\
\hline E5 & C --- C & No cambian \\
\hline E6 & C -- C & No cambian \\
\hline E7 & S --- S & No cambian \\
\hline E8 & S -- S & No cambian \\
\hline E9 & S --- S & Progresión Profesor \\
\hline E10 & T --- S & Progresión Profesor \\
\hline E11 & T -- CS & Progresión Profesor \\
\hline E12 & T --- S & Progresión Profesor \\
\hline E13 & TCA --- C & Progresión Profesor \\
\hline E14 & C --- S & \\
\hline
\end{tabular}




\section{Conclusiones}

Con relación al primer objetivo, la mayoría de los participantes han sido capaces de conceptualizar su roles como profesores en forma de metáforas. Tanto el texto escrito como los dibujos, se han mostrado como excelentes recursos para sintetizar el significado de las metáforas.

Con relación al segundo objetivo, los participantes han expresado las razones y explicaciones para identificarse con sus metáforas personales. En la mayor parte de los casos coinciden los significados del texto y del dibujo, pero en los casos en los que hay diferencias, es el dibujo el que mejor representa el significado de las metáforas.

Para el tercer objetivo hemos clasificado las metáforas personales de la muestra dentro de las categorías conductista-transmisiva, cognitiva-constructivista, situadas y auto-referenciadas. Al comparar las metáforas para el profesor de todos los participantes, antes y después de las prácticas de enseñanza se observa una gran disminución de las metáforas conductistas-transmisivas y un aumento de las cognitivasconstructivistas y de las situadas, manteniéndose un porcentaje similar para las autorreferenciadas. Sin embargo, como hemos indicado anteriormente, hay que tener en cuenta que el número de participantes fue mayor en el pretest que en postest.

Otro resultado significativo, coincidente con anteriores estudios (Mahlios et al., 2010; Mellado et al., 2012 y 2015), es que las metáforas no está asociadas a contenidos concretos, sino que son expresión de una visión general de la enseñanza y del rol del profesor, formada de sus propias experiencias como alumnos y de sus experiencias de enseñanza.

En cuanto a la evolución de los 14 participantes que realizaron el pre y el postest, 8 no cambiaron de categoría en sus metáforas, aunque alguno cambió las metáforas dentro de la misma categoría, lo que nos indica que para estos profesores las metáforas y sus modelos asociados están ya muy asentados al comienzo del Máster y la realización del mismo y de las prácticas no ha incidido en sus modelos. Sin embargo cinco futuros profesores cambiaron sus metáforas, realizando una progresión en sus metáforas de modelos didácticos centrados en el profesor y la enseñanza a otros más centrados en el alumnado y el aprendizaje.

Para el profesorado de Economía en formación, el conocimiento y la reflexión sobre sus propias metáforas, se ha mostrado como una buena herramienta para conceptualizar sus concepciones y sus roles docentes y, en algunos de ellos, para iniciar procesos de cambio en los mismos. Esta reflexión nos parece especialmente importante durante las prácticas de enseñanza, un periodo en el que se consolidan rutinas de enseñanza y en el que los futuros profesores están sometidos a una fuerte presión emocional.

Una implicación para continuar investigando en futuros trabajos, es que 
los resultados aportados están recogidos de lo que el profesor dice, pero no de la observación de lo que el profesor hace en el aula, y que sería necesario investigar para contrastar las metáforas y modelos declarados con los observados en la práctica del aula.

\section{Referencias bibliográficas}

ALARCÓN, P. y DÍAZ, C. (2014). El rol de la dinámica de fuerzas en la construcción reflexiva formal pasarse + dativo. Sintagma, $\mathrm{n}^{\circ}$ 26, 7-21.

ALARCÓN, P; DÍAZ, C; TAGLE, T; RAMOS, L. y QUINTANA, M. (2014). Metáforas para profesor y estudiante de pedagogía, en un grupo de estudiantes de pedagogía chilenos. Revista Actualidades Investigativas en Educación, $\mathrm{n}^{\circ} 14$ (2), 1-31. DOI: dx.doi.org/10.15517/aie.v14i2.14829

AUBUSSON, P. J; HARRISON, A. G. y RITCHIE, S. M. (2006). Metaphor and analogy. En P. J. AUBUSSON, A. G. HARRISON y S.T. RITCHIE (eds.), Metaphor and analogy in science education (1-9). Dordrecht: Springer.

BEN-PERETZ, M; MENDELSON, N. y KRON, F.W. (2003). How teachers in different educational contexts view their roles. Teaching and Teacher Education, $\mathrm{n}^{\circ} 19$ (2), 277-290. DOI: 10.1016/S0742-051X(02)00100-2

BOUJAOUDE, S. (2000). Conceptions of science teaching revealed by metaphors and answers to open-ended questions. Journal of Science Teacher Education, $\mathrm{n}^{\circ} 11$ (2), 173-186.
BUARAPHAN, K. (2011). Metaphorical roots of beliefs about teaching and learning science and their modifications in the standard based science teacher preparation programme. International Journal of Science Education, $\mathrm{n}^{\circ} 33$ (11), 1571-1595. DOI: 10.1080/09500693.2010.528462

BULLOUGH, JR. y ROVERT, V. (1991). Exploring personal teaching metaphors in preservice teacher education. Journal of Teacher Education, $\mathrm{n}^{\circ} 42$ (1), 43-51. DOI: 10.1177/002248719104200107

CAÑAS, F. y DE LA MONTAÑA, J. L. (2012). Reflexiones sobre el desarrollo profesional del profesorado de Economía. Tejuelo, $\mathrm{n}^{\circ}$ 1, 101-118.

CASSEL, D. y VINCENT, D. (2011). Metaphors reveal preservice elementary teachers' views of mathematics and science teaching. School Science and Mathematics, $\mathrm{n}^{\circ} 111$ (7), 319-324. DOI: 10.1111/j.19498594.2011.00094.x

CRAWFORD, B.A. (2000). Embracing the essence of inquiry: New roles for science teacher. Journal of Research in Science Teaching, $\mathrm{n}^{\circ} 37$ (9), 916937.DOI: 10.1002/1098-2736(200011) 37:9<916::aid-tea4>3.0.co; 2-2

DE BUSTOS, E. (2000). La metáfora. Ensayos Transdisciplinares. Madrid: Fondo de Cultura Económica.

DODD, S. D. (2002). Metaphors and meaning: A grounded cultural model of US entrepreneurship. Journal of Business Venturing, $\mathrm{n}^{\circ} 17$ (5), 519-535. DOI: 10.1016/S0883-9026(01)00072-6 
DUFFÉ, A. L. (2004). Reflexiones psicolingüísticas y didácticas sobre el estudio de metáforas y modismos. Didáctica (Lengua y Literatura), $\mathrm{n}^{\circ}$ 16, 33-44.

ESTEPA, J. y CUENCA, J. M. (2007). Las concepciones de los docentes y el desarrollo profesional: dos estudios desde la formación inicial en Ciencias Sociales. Investigación en la Escuela, $\mathrm{n}^{\circ} 61,85-98$.

FUKUDA, K. (2009). A comparative study of metaphors representing the US and Japanese economies. Journal of Pragmatics, $\mathrm{n}^{\circ} 41$ (9), 1693-1702. DOI: 10.1016/j.pragma.2008.12.005

GALLEGO, R. y PÉREZ, R. (2002). El problema del cambio en las concepciones de estudiantes de formación avanzada. Enseñanza de las Ciencias, $\mathrm{n}^{\circ} 20$ (3), 401-414.

GOSSELIN, C. y MEIXNER, E. (2015). Blank Canvas and Glass Ceiling: Using Visual Metaphors and Narratives to Examine Preservice Teacher Development. Multicultural Perspectives, $\mathrm{n}^{\mathrm{o}} 17$ (2), 73-80. DOI:10.1080/15 210960.2015.1022445

GURNEY, B. F. (1995). Tugboats and tennis games: Preservice conceptions of teaching and learning revealed through metaphors. Journal of Research in Science Teaching, $\mathrm{n}^{\mathrm{o}} 32$ (6), 569-583. DOI: 10.1002/ tea.3660320605

GUTIÉRREZ, P. y LUENGO, R. (2003). Orientación vocacional y género. Campo Abierto, $\mathrm{n}^{\circ} 23$ (1), 85-98.
HARGREAVES, A. (1996). Profesorado, cultura y modernidad. Madrid: Morata. HERRERA-SOLER, H. y White, M. (2012). Metaphors and Mills. Berlin: De Gruyter Mouton.

LAKOFF, G. y JOHNSON, M. (1986). Metáforas de la vida cotidiana. Madrid: Cátedra.

LEAVY,A. M; MCSORLEY, F.A. y BOTÉ, L. A. (2007). An examination of what metaphor construction reveals about the evolution of preservice teachers' beliefs about teaching and learning. Teaching and Teacher Education, $\mathrm{n}^{\circ} 23,1217-1233$. DOI: $10.1016 / \mathrm{j}$. tate.2006.07.016

LÓPEZ-LUENGO, M. A; TORRIGOEGIDO, L. M. y VALLÉS-RAPP, C. (2015). Qué metáforas personales definen al profesorado de Educación Infantil en formación. REICE, $\mathrm{n}^{\circ} 13$ (3), 37-56.

LORSBACH, A. W; TOBIN, K; BRISCOE, C. y LAMASTER, S. U. (1992). An interpretation of assessment methods in middle school science. International Journal of Science Education, $\mathrm{n}^{\circ} 14$ (3), 305-317. DOI:10.1080/0950069920140307

MADHAVARAM, S. y MCDONALD, R. E. (2010). Knowledge-based sales management strategy and the grafting metaphor: Implications for theory and practice. Industrial Marketing Management, $\mathrm{n}^{\circ} 39$ (7), 1078-1087. DOI: 10.1016/j.indmarman.2009.12.009

MAHLIOS, M; MASSENGILL-SHAW, 
D. y BARRY, A. (2010). Making sense of teaching through metaphors: a review across three studies. Teachers and Teaching: Theory and Practice, $\mathrm{n}^{\circ} 16$ (1), 49-71. DOI: 10.1080/13540600903475645

MARCOS, F. (1993). Diccionario básico de recursos expresivos. Badajoz: Universitas Editorial.

MARTÍNEZ, M. A; SAULEDA, N. y Huber, G. H. (2001). Metaphors as blueprints of thinking about teaching and learning. Teaching and Teacher Education, $\mathrm{n}^{\circ} 17$ (8), 965-977. DOI: 10.1016/S0742-051X(01)00043-9

MCCORKLE, S. y GAYLE, B. M. (2003). Conflict management metaphors: assessing everyday problem communication. The Social Science Journal, $\mathrm{n}^{\circ} 40$ (1), 137-142. DOI: 10.1016/ S0362-3319(02)00264-1

MELLADO, L; BERMEJO, M. L. y MELLADO, V. (2012). Personal metaphors of prospective secondary economics and science teachers. Asia Pacific Journal of Teacher Education, $\mathrm{n}^{\circ} 40$ (4), 395-408. DOI: 10.1080/1359866X.2012.724658

MELLADO, L; DE LA MONTAÑA, J. L; BORRACHERO, A. B. y BERMEJO (2014a). Las emociones en las metáforas personales de futuros profesores de educación de educación primaria y educación secundaria de economía. International Journal of Development and Educational Psychology, $\mathrm{n}^{\circ}$ 26 (2), 223-232.

MELLADO, L; LUENGO, M. R; DE LA MONTAÑA, J. L; BORRACHERO, A.
B. y BERMEJO, M. L. (2014b). Las metáforas emocionales de estudiantes de Psicopedagogía sobre el profesor y el aprendizaje. Campo Abierto. Revista de Educación, n ${ }^{\circ} 33$ (1), 153170.

MELLADO, L; LUENGO, M. R; DE LA MONTAÑA, J. L. y BERMEJO, M. L. (2015). Emotions in Personal Metaphors of Prospective Secondary Economics, Science, and Psychopædagogy Teachers. En A. TAVIDZE (ed.), Progress in Economics Research, (2746). New York: Nova Science Publishers.

MELLADO, V; RUIZ, C; BERMEJO, M. L. y JIMÉNEZ, R. (2006). Contributions from the philosophy of science to the education of science teachers. Science and Education, $\mathrm{n}^{\circ} 15$ (5), 419445.

MORGAN, G. (1980). Paradigm, metaphors, and puzzle solving in organization theory. Administrative Science Quarterly, $\mathrm{n}^{\circ} 25$ (4), 605-622. DOI: 10.2307/2392283.

PAAVOLA, S. y HAKKARAINEN, K. (2005). The knowledge creation metaphor. An emergent epistemological approach to learning. Science and Education, $\mathrm{n}^{\circ} 14,535-557$. DOI: 10.1007/s11191-004-5157-0.

PINNEGAR, S; MANGELSON, J; REED, M. y GROVES, S. (2011). Exploring preservice teachers' metaphor plotlines. Teaching and Teacher Education, n ${ }^{\circ}$ 27, 639-647.

REBOLLO, M. A; GARCÍA, R; BARRAGÁN, R; BUZÓN, O. y VEGA, L. 
(2008). Las emociones en el aprendizaje online. Relieve, $\mathrm{n}^{\circ} 14$ (1).

REBOLLO, M.A; JIMÉNEZ, R; SABUCO, A. y VEGA, L. (2013). Metáforas que usan las profesoras en los procesos de cambio educativo con perspectiva de género. Profesorado: Revista de curriculum y formación del profesorado, $\mathrm{n}^{\mathrm{o}} 17$ (1), 43-56.

RENÉ, T. y STOFFLETT, R. T. (1996). Metaphor development by secondary teachers enrolled in graduate teacher education. Teaching and Teacher Education, $\mathrm{n}^{\circ} 12$ (6), 577-589. DOI: 10.1016/s0742-051x(96)00002-9

RITCHIE, S. M. (1994). Metaphor as a tool for constructivist science teaching. International Journal of Science Education, $\mathrm{n}^{\circ} 16$ (3), 293-303. DOI: 10.1080/0950069940160304

RUSSELL, T. y HRYCENKO, M. (2006). The role of Metaphor in a new science teacher's learning from experience. En P. J. AUBUSSON, A. G; HARRISON y S. T. RITCHIE (eds.), Metaphor and analogy in science education (131-142). Dordrecht: Springer.

SABAN, A. (2010). Prospective teachers' metaphorical conceptualizations of learner. Teaching and Teacher Education, $\mathrm{n}^{\circ} 26$ (2), 290-305.

SABAN, A; KOCBEKER, B. N. y SABAN, A. (2007). Prospective teachers' conceptions of teaching and learning revealed through metaphor analysis. Learning and Instruction, $\mathrm{n}^{\circ} 17,123-139$.

SEFEROGLU, G; KORKMAZGIL, S. y ÖLÇÜ, Z. (2009). Gaining insights into teachers' ways of thin- king via metaphors. Educational Studies, $\mathrm{n}^{\circ} 35$ (3), 323-335. DOI: 10.1080/03055690802648135

SERRANO, J. A. A. y POZO, J. I. (2006). De fotógrafos a directores de orquesta: las metáforas desde las que los profesores conciben el aprendizaje. En Nuevas formas de pensar la enseñanza y el aprendizaje: las concepciones de profesores y alumnos (265288). Barcelona: Graó.

SEUNG, E., PARK, S. y NARAYAN, R. (2011). Exploring elementary preservice teachers' beliefs about science teaching and learning as revealed in their metaphor writing. Journal of Science Education and Technology, $\mathrm{n}^{\circ}$ 20 (6), 703-714. DOI: 10.1007/s10956010-9263-2.

SHAW, D. M; BARRY, A. y MAHLIOS, M. (2008). Preservice teachers' metaphors of teaching in relation to literacy beliefs. Teachers and Teaching: Theory and Practice, $\mathrm{n}^{\circ} 14$ (1), 35-50. DOI: $10.1080 / 13540600701837632$.

SILLMAM, K. y DANA, T. M. (2001). Metaphor: a tool for promoting prospective elementary teachers' participation in science teacher learning community. Journal of Science Teacher Education, $\mathrm{n}^{\circ} 12$ (2), 87-106.

TANNEHILL, D. y MACPHAIL, A. (2014). What examining teaching metaphors tells us about pre-service teachers' developing beliefs about teaching and learning. Physical Education and Sport Pedagogy, $\mathrm{n}^{\circ} 19$ (2), 149-163. DOI:10.1080/17408989.201 2.732056 
TEICHERT, T ; VON WARTBURG, I. y BRATERMAN, R. (2006). Tacit meaning in disguise: Hidden metaphors in new product development and market making. Business Horizons, n 49 (6), 451-461. DOI: 10.1016/j.bushor.2006.02.006

THOMAS, L. C. y BEAUCHAMP, C. (2011). Understanding new teachers' professional identities through metaphor. Teaching and Teacher Education, $\mathrm{n}^{\circ} 27$ (4), 762-769. DOI: 10.1016/j. tate.2010.12.007

TOBIN, K. y LAMASTER, S.U. (1995). Relationships between metaphors, beliefs, and actions in a context of science curriculum. Journal of Research in Science Teaching, $\mathrm{n}^{\circ} 32$ (3), 225-242. DOI: 10.1002/tea.3660320304

TOBIN, K. y TIPPINS, D. J. (1996). Metaphors as seeds for conceptual change and the improvement of science education. Science Education, $\mathrm{n}^{\circ} 80$ (6), 711-730. DOI: 10.1002/(SICI)1098$237 \mathrm{X}(199611) 80: 6<711:$ :AID SCE5>3.0.CO;2-M

TOBIN, K; TIPPINS, D. J. y GALLARD, A. J. (1994). Research on instructional strategies for teaching science. En D. Gabel (ed.), Handbook of Research on Science Teaching and Learning (3-44). New York: MacMillan.

TRAVÉ, G. (1998). La investigación en didáctica de las ciencias sociales. Huelva: Publicaciones de la Universidad de Huelva.
TRAVÉ, G. y MOLINA, J. A. (2014). Concepciones y prácticas del profesorado de economía sobre el uso de materiales curriculares: un análisis exploratorio. Enseñanza de las Ciencias Sociales, $\mathrm{n}^{\circ}$ 13, 71-83.

VOLKMANN, M. J. y ANDERSON, M. A. (1998). Creating professional identity: dilemmas and metaphors of a first-year chemistry teacher. Science Education, $\mathrm{n}^{\circ} 82$ (3), 293-310. DOI: $10.1002 /(S I C I) 1098-$ $237 \mathrm{X}(199806) 82: 3<293:$ :AID SCE1>3.0.CO;2-7

WALTERS, M. y YOUNG, J. J. (2008). Metaphors and accounting for stock options. Critical Perspectives on Accounting, $\mathrm{n}^{\circ} 19$ (5), 805-833. DOI: 10.1016/j.cpa.2006.10.004

WEINER, B. (1991). Metaphors in Motivation and Attribution. American Psychologist, $\mathrm{n}^{\circ} 46$ (9), 921-930.

WILLISON, J. W. y TAYLOR, P. Ch. (2006). Complementary epistemologies of science teaching. En P. J. Aubusson, A. G. Harrison y S. T. Ritchie (eds.), Metaphor and analogy in science education (25-36). Dordrecht: Springer. DOI: 10.1007/1-4020-38305_3

ZHAO,H;COOMBS,S.yZHOU,X.(2011). Developing professional knowledge about teachers through metaphor research: facilitating a process of change. Teacher Development, $\mathrm{n}^{\circ} 14$ (3), 381-395. DOI: 10.1080/13664530 